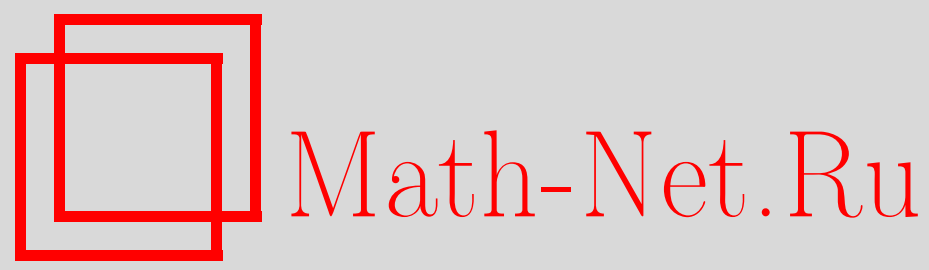

В. П. Черкашин, О точных деформациях пуассоновых модулей, Матем. заметки, 1996, том 59, выпуск 4, 633-637

DOI: https://doi.org/10.4213/mzm1758

Использование Общероссийского математического портала MathNet.Ru подразумевает, что вы прочитали и согласны с пользовательским соглашением

http://www. mathnet.ru/rus/agreement

Параметры загрузки:

IP : 54.197 .130 .99

26 апреля 2023 г., 06:03:34

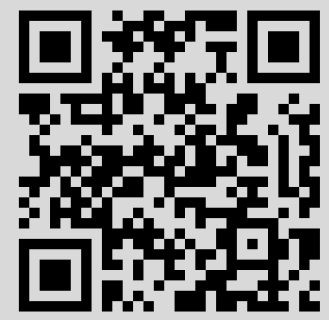




\section{О ТОЧНЫХ ДЕФОРМАЦИЯХ ПУАССОНОВЫХ МОДУЛЕЙ}

\section{В.П. Черкашин}

Известный факт состоит в том, что 2-коцикл задает, вообще говоря, лишш инфинитезимальную деформацию алгебраической структуры: определяющие тождества гарантированы только по модулю второго порядка. Для разложимых в тензорное произведение пуассоновых алгебр в [1] был сконструирован 2-коцикл, деформация при помощи которого является точной, т.е. деформированный коммутатор удовлетворяет тождеству Якоби modulo 0. Иными словами, найденный 2-коцикл есть решение уравнения деформаций [2].

В этой заметке построены двухпараметрические семейства точных деформаций (согласованные лиевы структуры, ср. [3, с. 32]) тензорных произведений пуассоновых модулей [1], [4], их гомотопий и частных, сопровождающие соответствующие деформации пуассоновых алгебр.

Основное кольцо обозначим $K$.

1. Комплекс модульных коцепей. Пусть $\mathfrak{g}-K$-алгебра Ли, $\mathfrak{M}-\mathfrak{g}$-модуль, $\left(C^{\bullet}(\mathfrak{g} ; \mathfrak{g}), \delta\right)$ - комплекс коцепей алгебры $\mathfrak{g}$ со значениями в $\mathfrak{g}, C_{\mathfrak{M}}^{0}(\mathfrak{g} \mid \mathfrak{M}):=\mathfrak{M}$, при $p \geqslant 1 C_{\mathfrak{M}}^{p}(\mathfrak{g} \mid \mathfrak{M}):=K$-модуль полилинейных отображений $\mathfrak{g}^{\times(p-1)} \times \mathfrak{M} \rightarrow \mathfrak{M}$, $\left(x_{1}, \ldots, x_{p-1}, m\right) \mapsto \psi\left(x_{1}, \ldots, x_{p-1} ; m\right) \in \mathfrak{M}, x_{1}, \ldots, x_{p-1} \in \mathfrak{g}, m \in \mathfrak{M}$, кососимметрических по $x_{1}, \ldots, x_{p-1}, C^{p}(\mathfrak{g} \mid \mathfrak{M}):=C^{p}(\mathfrak{g} ; \mathfrak{g}) \oplus C_{\mathfrak{M}}^{p}(\mathfrak{g} \mid \mathfrak{M}), p \geqslant 0$, $C^{\bullet}(\mathfrak{g} \mid \mathfrak{M}):=\bigoplus_{p \geqslant 0} C^{p}(\mathfrak{g} \mid \mathfrak{M})($ cp. $[2, \S 10])$,

$$
\begin{aligned}
& \Delta(\varphi, \psi):=\left(\delta_{\mathfrak{g}}(\varphi, \psi), \delta_{\mathfrak{M}}(\varphi, \psi)\right), \quad \delta_{\mathfrak{g}}(\varphi, \psi):=\delta(\varphi), \\
& \delta_{\mathfrak{M}}(\varphi, \psi)\left(x_{1}, \ldots, x_{p} ; m\right) \\
& :=\sum_{1 \leqslant i \leqslant p}(-1)^{i+1}\left[x_{i}, \psi\left(x_{1}, \ldots, \widehat{x}_{i}, \ldots, x_{p} ; m\right)\right]+(-1)^{p+1}\left[\varphi\left(x_{1}, \ldots, x_{p}\right), m\right] \\
& \quad+\sum_{1 \leqslant i<j \leqslant p}(-1)^{i+j} \psi\left(\left[x_{i}, x_{j}\right], x_{1}, \ldots, \widehat{x}_{i}, \ldots, \widehat{x}_{j}, \ldots, x_{p} ; m\right) \\
& +\sum_{1 \leqslant i \leqslant p}(-1)^{i} \psi\left(x_{1}, \ldots, \widehat{x}_{i}, \ldots, x_{p} ;\left[x_{i}, m\right]\right), \quad(\varphi, \psi) \in C^{p}(\mathfrak{g} \mid \mathfrak{M}) .
\end{aligned}
$$

(Здесь и далее, ради графической простоты, приходится использовать одни и те же квадратные скобки для коммутаторов и лиевых действий алгебр на модулях, но смысл при этом однозначно определен элементами, участвующими в композициях.)

ЗАмЕч АниЕ. В специальном случае, когда $\mathfrak{g}$-коммутаторная алгебра $V^{(-)}$[5, c. 181, 239] левосимметричной алгебры (алгебры Винберга) $V[6$, c. 322], $\mathfrak{M}=V$, $V^{(-)}$действует на $V$ левыми умножениями алгебры $V, \delta_{\mathfrak{M}}(\psi, \psi)$ совпадает с конструкцией кограницы для построенного в [7, с. 94] комплекса.

ПРЕДЛОЖЕНИЕ. $\Delta \circ \Delta=0$.

ДоКАЗАТЕЛЬСТВо. Достаточно вычислить $\delta_{\mathfrak{M}}\left(\delta(\varphi), \delta_{\mathfrak{M}}(\varphi, \psi)\right)$.

Пусть $\mathfrak{g}$ - пуассонова $K$-алгебра, $\mathfrak{M}-\mathfrak{g}$-модуль, $C^{0}(\mathfrak{g} \mid \mathfrak{M})$, при $p \geqslant 1$ $D^{p}(\mathfrak{g} \mid \mathfrak{M}):=K$-подмодуль в $C^{p}(\mathfrak{g} \mid \mathfrak{M})$, состоящий из таких $(\varphi, \psi)$, что $\varphi-p$-диффференцирование $\left[8\right.$, c. 89] ассоциативной алгебры $\mathfrak{g}$, отображение $\left(x_{1}, \ldots, x_{p-1}\right) \mapsto$ $\psi\left(x_{1}, \ldots, x_{p-1} ; m\right)$ есть $(p-1)$-диффференцирование ассоциативной алгебры $\mathfrak{g}, \mathrm{a}$ 
отображение $m \mapsto \psi\left(x_{1}, \ldots, x_{p-1} ; m\right)$ есть диффференцирование модуля $\mathfrak{M}$ над дифференцированием $x \mapsto \varphi\left(x_{1}, \ldots, x_{p-1}, x\right)[8$, c. 201]:

$$
\begin{gathered}
\psi\left(x_{1}, \ldots, x_{p-1} ; x \cdot m\right)=\varphi\left(x_{1}, \ldots, x_{p-1}, x\right) \cdot m+x \cdot \psi\left(x_{1}, \ldots, x_{p-1} ; m\right) \\
x_{1}, \ldots, x_{p-1}, x \in \mathfrak{g}, \quad m \in \mathfrak{M} .
\end{gathered}
$$

Положим $D^{\bullet}(\mathfrak{g} \mid \mathfrak{M}):=\bigoplus_{p \geqslant 0} D^{p}(\mathfrak{g} \mid \mathfrak{M})$.

Лемма. Дифференииал $\Delta$ допускает ограничение на $D^{\bullet}(\mathfrak{g} \mid \mathfrak{M})$.

ДокАЗАТЕЛЬство. Непосредственная проверка.

Коцепи, коциклы и т.д. комплекса $\left(D^{\bullet}(\mathfrak{g} \mid \mathfrak{M}), \Delta\right)$ будем называть модульными $(\mathfrak{g}, \mathfrak{M})$-кочепями, -кочиклами и т.д.

2. Частные. Пусть $A$ - коммутативная ассоциативная унитальная $K$-алгебра, $M$ - унитальный $A$-модуль [9, с. 27], $Q$ - мультипликативный подмоноид [10, c. 73] в алгебре $A, Q^{-1} A-K$-алгебра частных, $Q^{-1} M-Q^{-1} A$-модуль частных, кан $A: A \rightarrow Q^{-1} A, x \mapsto x / 1$, кан $M: M \rightarrow Q^{-1} M, m \mapsto m / 1$ - канонические морфизмы $[9$, с. 49$]$.

Лемма. Пусть $p \in \mathbb{N}, \varphi: A^{\times p} \rightarrow M-p$-дифференцирование, $\psi:$ $A^{\times(p-1)} \times M \rightarrow M-(p-1)$-дифференчирование алгебры $A$, дифферениирование модуля $M$ над дифферениированием $x \mapsto \varphi\left(x_{1}, \ldots, x_{p-1}, x\right)$, $x_{1}, \ldots, x_{p-1}, x \in A$. Тогда

1) существует и притом единственное $p$-дифференцирование $Q^{-1} \varphi$ : $\left(Q^{-1} A\right)^{\times p} \rightarrow Q^{-1} M$ maкое, что $Q^{-1} \varphi \circ$ кан $_{A}^{\times p}=$ кан $_{M} \circ \varphi ;$

2) существует и притом единственное $(p-1)$-дифферениирование алгебры $Q^{-1} A$, дифференцирование $Q^{-1} A$-модуля $Q^{-1} M$ над дифференцированием

$$
\begin{gathered}
\frac{x^{0}}{x^{1}} \mapsto Q^{-1} \varphi\left(\frac{x_{1}^{0}}{x_{1}^{1}}, \ldots, \frac{x_{p-1}^{0}}{x_{p-1}^{1}}, \frac{x^{0}}{x^{1}}\right), \\
x_{1}^{0}, \ldots, x_{p-1}^{0}, x^{0} \in A, \quad x_{1}^{1}, \ldots, x_{p-1}^{1}, x^{1} \in Q, \\
Q^{-1} \psi:\left(Q^{-1} A\right)^{\times(p-1)} \times Q^{-1} M \rightarrow Q^{-1} M
\end{gathered}
$$

такое, ито $Q^{-1} \psi \circ\left(\right.$ кан $_{A}^{\times}(p-1) \times$ кан $\left._{M}\right)=$ кан $_{M} \circ \psi$;

3) если $\varphi, \psi$ кососимметричны, то и $Q^{-1} \varphi, Q^{-1} \psi$ кососимметричны.

ДоКАЗАТЕльСТВо. Если отображение $Q^{-1} \varphi$ существует, то оно определено однозначно:

$$
Q^{-1} \varphi\left(\frac{x_{1}^{0}}{x_{1}^{1}}, \ldots, \frac{x_{p}^{0}}{x_{p}^{1}}\right)=\sum_{\left(\varepsilon_{1}, \ldots, \varepsilon_{p}\right)}(-1)^{\varepsilon_{1}+\cdots+\varepsilon_{p}} x_{1}^{1+\varepsilon_{1}} \cdots x_{p}^{1+\varepsilon_{p}} \cdot \frac{\varphi\left(x_{1}^{\varepsilon_{1}}, \ldots, x_{p}^{\varepsilon_{p}}\right)}{\left(x_{1}^{1} \cdots x_{p}^{1}\right)^{2}}
$$

где $x_{1}^{0}, \ldots, x_{p}^{0} \in A, x_{1}^{1}, \ldots, x_{p}^{1} \in Q$, суммирование - по всем $\left(\varepsilon_{1}, \ldots, \varepsilon_{p}\right) \in$ $(\mathbb{Z} / \mathbb{Z} \cdot 2)^{\times p}$, а проверка показьвает, что эта композиция не зависит от выбора 
представителей классов дробей и корректно определяет искомое отображение. Аналогично,

$$
\begin{aligned}
Q^{-1} \psi\left(\frac{x_{1}^{0}}{x_{1}^{1}}, \ldots,\right. & \left.\frac{x_{p-1}^{0}}{x_{p-1}^{1}} ; \frac{m}{x_{p}^{1}}\right)=\sum_{\left(\varepsilon_{1}, \ldots, \varepsilon_{p-1}\right)}(-1)^{\varepsilon_{1}+\cdots+\varepsilon_{p-1}} x_{1}^{1+\varepsilon_{1}} \cdots x_{p-1}^{1+\varepsilon_{p-1}} \\
& \frac{x_{p}^{1} \cdot \psi\left(x_{1}^{\varepsilon_{1}}, \ldots, x_{p-1}^{\varepsilon_{p-1}} ; m\right)-\varphi\left(x_{1}^{\varepsilon_{1}}, \ldots, x_{p-1}^{\varepsilon_{p-1}}, x_{p}^{1}\right) \cdot m}{\left(x_{1}^{1} \cdots x_{p}^{1}\right)^{2}}, \quad m \in M .
\end{aligned}
$$

Пусть $\mathfrak{g}$ - пуассонова $K$-алгебра, $Q$ - мультипликативный подмоноид в ассоциативной алгебре $\mathfrak{g}$. Тогда ассоциативная $K$-алгебра $Q^{-1} g$ имеет и притом единственную структуру пуассоновой алгебры такую, что $[x / 1, y / 1]=[x, y] / 1, x, y \in \mathfrak{g}[11]$.

Пусть $\mathfrak{M}$ - пуассонов $\mathfrak{g}$-модуль, $D^{\bullet}(\mathfrak{g} ; \mathfrak{M})$ - комплекс коцепей алгебры $\mathfrak{g}$ со значениями в $\mathfrak{M}[1]$, [4].

TЕОремА. Модуль $Q^{-1} \mathfrak{M}$ над ассочиативной алгеброй $Q^{-1} \mathfrak{g}$ имеет $u$ притом единственную структуру модуля над пуассоновой алгеброй $Q^{-1} \mathfrak{g}$ такую, что $[x / 1, m / 1]=[x, m] / 1, x \in \mathfrak{g}, m \in \mathfrak{M}$.

ДОКАЗАТЕЛЬСТВО. В силу предыдущей леммы

$$
\begin{gathered}
{\left[\frac{x}{q}, \frac{m}{r}\right]=\frac{q r \cdot[x, m]-q[x, r] \cdot m-x r \cdot[q, m]+x[q, r] \cdot m}{q^{2} r^{2}},} \\
x \in \mathfrak{g}, \quad q, r \in Q, \quad m \in \mathfrak{M} .
\end{gathered}
$$

Рутинное вычисление обнаруживает, что эта композиция является лиевьм действием пуассоновой алгебры $Q^{-1} \mathfrak{g}$ на $Q^{-1} \mathfrak{M}$.

Теорема. 1) Прямая сумма отображсений

$$
D^{p}(\mathfrak{g} ; \mathfrak{M}) \ni \varphi \mapsto Q^{-1} \varphi \in D^{p}\left(Q^{-1} \mathfrak{g} ; Q^{-1} \mathfrak{M}\right), \quad p \geqslant 0
$$

есть морфизм комплексов.

2) Прямая сумма отобрахсений

$$
D^{p}(\mathfrak{g} \mid \mathfrak{M}) \ni(\varphi, \psi) \mapsto\left(Q^{-1} \varphi, Q^{-1} \psi\right) \in D^{p}\left(Q^{-1} \mathfrak{g} \mid Q^{-1} \mathfrak{M}\right), \quad p \geqslant 0,
$$

есть морфизм комплексов.

ДоКАЗАТЕльство. Вычисления показьвают, что отображения $\varphi \mapsto Q^{-1} \varphi$ и $(\varphi, \psi) \mapsto\left(Q^{-1} \varphi, Q^{-1} \psi\right)$ перестановочны с дифференциалами.

3. Гомотопии. Пусть $\mathfrak{g}$ - пуассонова $K$-алгебра, $\mathrm{hmt}(\mathfrak{g})$ - алгебра гомотопий алгебры $\mathfrak{g}[12], \mathfrak{M}-\mathfrak{g}$-модуль. Обозначим $\mathrm{hmt}(\mathfrak{g} ; \mathfrak{M})$ множество элементов $t \in \operatorname{hmt}(\mathfrak{g})$, для которых вьполнено тождество $\operatorname{cycl}_{(x, y, t)}[x, y] \cdot[t, m]=0, x, y \in \mathfrak{g}$, $m \in \mathfrak{M}$. Имеют место очевидные включения $\operatorname{cen}(\mathfrak{g}) \cap \operatorname{cen}_{\mathfrak{g}}(\mathfrak{M}) \leqslant \operatorname{hmt}(\mathfrak{g} ; \mathfrak{M}) \leqslant$ $\operatorname{hmt}(\mathfrak{g}) \leqslant \mathfrak{g}$, где $\operatorname{cen}(\mathfrak{g})$ - центр алгебры Ли $\mathfrak{g}, \operatorname{cen}_{\mathfrak{g}}(\mathfrak{M})$ - централизатор модуля $\mathfrak{M}$ в алгебре Ли $\mathfrak{g}$. Пусть $\mathfrak{g}(t)$ - гомотопия алгебры $\mathfrak{g}$ при помощи элемента $t \in \operatorname{hmt}(\mathfrak{g})[12]$.

TЕоремА. 1) $\operatorname{hmt}(\mathfrak{g} ; \mathfrak{M})$ - ассочиативная подалгебра и лиев идеал в пуассоновой алгебре $\mathfrak{g}$.

2) Для любого $t \in \operatorname{hmt}(\mathfrak{g} ; \mathfrak{M})$ модуль $\mathfrak{M}$ над ассоииативной алгеброй $\mathfrak{g}$ является модулем над пуассоновой алгеброй $\mathfrak{g}(t)$ относительно действия $(x, m) \mapsto t \cdot[x, m], x \in \mathfrak{g}, m \in \mathfrak{M}$ 
ДоКАЗАТЕЛЬСтво. 1) Использовать тождество, аналогичное тому, которое применено в доказательстве предложения п. 1 [12].

2) Непосредственная проверка.

4. Деформации. Пусть $\mathfrak{g}$ и $\mathfrak{h}$-пуассоновы $K$-алгебры, $Q$ и $R$-мультипликативные подмоноиды в ассоциативных алгебрах $\mathfrak{g}$ и $\mathfrak{h}$ соответственно, $\mathfrak{M}-\mathfrak{g}$-модуль, $\mathfrak{N}-\mathfrak{h}$-модуль. Тензорные произведения пуассоновых алгебр и модулей над ними считаем наделенными каноническими пуассоновыми структурами [1].

Пусть $\theta: \mathfrak{g} \otimes \mathfrak{h} \rightarrow \mathfrak{g} \otimes \mathfrak{h}$ и $\lambda: \mathfrak{M} \otimes \mathfrak{h} \rightarrow \mathfrak{M} \otimes \mathfrak{h}-$ такие эндоморфизмы $K$-модулей, что $\theta-2$-дифференцирование пуассоновых алгебр $[1, \S 4]$, и для любых $x, y \in \mathfrak{g}$, $u, v \in \mathfrak{h}, m \in \mathfrak{M}$, выполнены следующие условия (*):

$$
\begin{gathered}
\lambda(x \cdot m \otimes u)=\theta(x \otimes u) \cdot(m \otimes 1)+(x \otimes 1) \cdot \lambda(m \otimes u), \\
\lambda(m \otimes u v)=(1 \otimes v) \cdot \lambda(m \otimes u)+(1 \otimes u) \cdot \lambda(m \otimes v), \\
\lambda([x, m] \otimes u)=[\theta(x \otimes u), m \otimes 1]+[x \otimes 1, \lambda(m \otimes u)], \\
\lambda(m \otimes[u, v])=[1 \otimes u, \lambda(m \otimes v)]-[1 \otimes v, \lambda(m \otimes u)], \\
\begin{aligned}
\theta((x \otimes 1) \theta(y \otimes u))- & (x \otimes 1) \theta(\theta(y \otimes u)) \\
& =\theta(\theta(x \otimes u)(y \otimes 1))-\theta(\theta(x \otimes u))(y \otimes 1), \\
\theta((1 \otimes u) \theta(x \otimes v))- & (1 \otimes u) \theta(\theta(x \otimes v)) \\
& =\theta((1 \otimes v) \theta(x \otimes u))-(1 \otimes v) \theta(\theta(x \otimes u)), \\
\lambda((x \otimes 1) \cdot \lambda(m \otimes u))- & (x \otimes 1) \cdot \lambda(\lambda(m \otimes u)) \\
& =\lambda(\theta(x \otimes u) \cdot(m \otimes 1))-\theta(\theta(x \otimes u)) \cdot(m \otimes 1), \\
\lambda((1 \otimes u) \cdot \lambda(m \otimes v))- & (1 \otimes u) \cdot \lambda(\lambda(m \otimes v)) \\
= & \lambda((1 \otimes v) \cdot \lambda(m \otimes u))-(1 \otimes v) \cdot \lambda(\lambda(m \otimes u)) .
\end{aligned}
\end{gathered}
$$

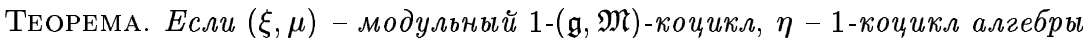
$\mathfrak{h}$ со значениями в $\mathfrak{h}$, то $\theta_{1}:=\xi \otimes \eta$ и $\lambda_{1}:=\mu \otimes \eta$ удовлетворяют условияM $(*)$.

ДокАЗАТЕЛЬство. Непосредственная проверка.

СледСтвиЕ. Эндоморфизмы $\theta_{2}:=Q^{-1} \xi \otimes R^{-1} \eta \in \operatorname{end}_{K}\left(Q^{-1} \mathfrak{g} \otimes R^{-1} \mathfrak{h}\right) u$ $\lambda_{2}:=Q^{-1} \mu \otimes R^{-1} \eta \in$ end $_{K}\left(Q^{-1} \mathfrak{M} \otimes R^{-1} \mathfrak{h}\right)$ удовлетворяют условиям (*).

ДоКАЗАТЕЛЬСТВо. Согласно второй теореме п. 2, отображения перехода к частным сохраняют коциклы.

TEOPEMA. Пусть $a, b \in K$.

1) Ассочиативная алгебра $\mathfrak{g} \otimes \mathfrak{h}$ с коммутатором $[x \otimes u, y \otimes v]_{(a, b)}:=$ $a[x \otimes u, y \otimes v]+b(\theta(x \otimes v)(y \otimes u)-(x \otimes v) \theta(y \otimes u))$ является пуассоновой алгеброй.

2) Если, кроме того, пуассонова алгебра $\mathfrak{h}$ тривиальна [1], $t \in \mathfrak{h}, u$ $\operatorname{cycl}_{(x, y, z)}([x, y] \otimes 1) \theta(z \otimes t)=0$ тохсдественно по $x, y, z \in \mathfrak{g}$, то ассоииативная алгебра $\mathfrak{g} \otimes \mathfrak{h}$ с коммутатором $[x \otimes u, y \otimes v](t)_{(a, b)}:=$ $a[x, y] \otimes t u v+b(\theta(x \otimes v)(y \otimes u)-(x \otimes v) \theta(y \otimes u))$ является пуассоновой алгеброй.

3) Модуль $\mathfrak{M} \otimes \mathfrak{h}$ над ассоииативной аләеброй $\mathfrak{g} \otimes \mathfrak{h}$ является модулем над пуассоновой алгеброй утверждения 1) относительно действия $[x \otimes u, m \otimes v]_{(a, b)}:=a[x \otimes u, m \otimes v]+b(\theta(x \otimes v) \cdot(m \otimes u)-(x \otimes v) \cdot \lambda(m \otimes u))$. 
4) Если выполнены условия утверждения 2) и, кроме того, $([x, y] \otimes 1)$. $\lambda(m \otimes t)+\theta(x \otimes t) \cdot([y, m] \otimes 1)-\theta(y \otimes t) \cdot([x, m] \otimes 1)=0$ тохдественно по $x, y \in \mathfrak{g}, m \in \mathfrak{M}$, mо модуль $\mathfrak{M} \otimes \mathfrak{h}$ над ассоииативной алгеброй $\mathfrak{g} \otimes \mathfrak{h}$ является модулем над пуассоновой алгеброй утверждения 2) относительно действия $[x \otimes u, m \otimes v](t)_{(a, b)}:=a[x, m] \otimes t u v+b(\theta(x \otimes v)$. $(m \otimes u)-(x \otimes v) \cdot \lambda(m \otimes u))$.

5) Пусть $(\xi, \mu)-м о д у л ь н ы и ̆ ~ 1-(\mathfrak{g}, \mathfrak{M})-\kappa о ч и к \Omega,(\eta, \nu)-м о д у л ь н ы и ̆ ~ 1-(\mathfrak{h}, \mathfrak{N})$ коиикл, $\theta=\xi \otimes \eta$. Тогда модуль $\mathfrak{M} \otimes \mathfrak{N}$ над ассоииативной алгеброй $\mathfrak{g} \otimes \mathfrak{h}$ является модулем над пуассоновой алгеброй утвержсдения 1) относительно действия $[x \otimes u, m \otimes n]_{(a, b)}:=a[x \otimes u, m \otimes n]+b(\xi(x) \cdot m \otimes$ $u \cdot \nu(n)-x \cdot \mu(m) \otimes \eta(u) \cdot n)$.

6) Если выполнены условия утверждений 2) и 5), $\mathfrak{N}$ - тривиальный $\mathfrak{h}$-модуль и, кроме того, $[x, y] \cdot \mu(m)+\xi(x) \cdot[y, m]-\xi(y) \cdot[x, m]=0$, mo модуль $\mathfrak{M} \otimes \mathfrak{N}$ над ассоииативной алгеброй $\mathfrak{g} \otimes \mathfrak{h}$ является модулем над пуассоновой алгеброй утверждения 2) относительно действия $[x \otimes u, m \otimes n](t)_{(a, b)}:=a[x, m] \otimes t u \cdot n+b(\xi(x) \cdot m \otimes u \cdot \nu(n)-x \cdot \mu(m) \otimes \eta(u) \cdot n)$.

ДоказАтЕльство. 1) Следствие теоремы [1, § 4]. 2)-6) Рутинные вычисления.

ЗАмЕчАния. а) Каноническим пуассоновым структурам соответствует $(a, b)=(1,0)$.

б) Дополнительные условия утверждений 2), 4) (для случая $\theta=\xi \otimes \eta, \lambda=\mu \otimes \eta$ ) и 6) на $\theta, \lambda, \xi, \mu$ выполнены, если $(\xi, \mu)$-модульная 1 - $(\mathfrak{g}, \mathfrak{M})$-кограница: $\xi(x)=[x, h]$, $\mu(m)=-[h, m]$ для некоторого $h \in \operatorname{hmt}(\mathfrak{g} ; \mathfrak{M})$.

в) Модульные 1 - $\left(Q^{-1} \mathfrak{g}, Q^{-1} \mathfrak{M}\right)$-коцикл $\left(Q^{-1} \xi, Q^{-1} \mu\right)$ и 1-( $\left.R^{-1} \mathfrak{h}, R^{-1} \mathfrak{N}\right)$-коцикл $\left(R^{-1} \eta, R^{-1} \nu\right)$ определяют деформацию модуля $Q^{-1} \mathfrak{M} \otimes R^{-1} \mathfrak{N}$ согласно 5), а если $(\xi, \nu)$ - модульная 1-(g, M)-кограница, то - и согласно 6), так как если $h \in \operatorname{hmt}(\mathfrak{g} ; \mathfrak{M})$, то $h / 1 \in \operatorname{hmt}\left(Q^{-1} \mathfrak{g}, Q^{-1} \mathfrak{M}\right)$.

Москва

Поступило 01.08 .95

\section{СПИСОК ЦИТИРОВАННОЙ ЛИТЕРАТУРЫ}

1. Черкашин В. П. // Матем. заметки. 1993. Т. 54. № 3. С. 141-151. 2. Nijenhuis A., Richardson R. W. // J. Math. Mech. 1967. V. 17. № 1. P. 89-105. 3. Карасев М. В., Маслов В. П. Нелинейные скобки Пуассона. Геометрия и квантование. M.: Наука, 1991. 4. Krasil'shchik I. S. // Lect. Notes in Math. 1988. V. 1334. Р. 79-110. 5. Шестаков И. П., Кузьмин Е. Н. // Итоги науки и техн. Соврем. пробл. матем. Фундамент. направления. Т. 57. М.: ВИНИТИ, 1990. С. 179-266. 6. Трофимов В. В. Введение в геометрию многообразий с симметриями. М.: МГУ, 1989. 7. Nijenhuis A. // Nieuw Archief voor Wiskunde. 1969. D. 17. № 2. Р. 87-108. 8. Виноградов А.М., Красильщик И. С., Лычагин В. В. Введение в геометрию нелинейных дифференциальных уравнений. М.: Наука, 1986. 9. Атья М., Макдональд И. Введение в коммутативную алгебру. М.: Мир, 1972. 10. Фейс К. Алгебра: кольца, модули и категории. Т. 1. М.: Мир, 1977. 11. Berger R. // C. R. Acad. Sci. Paris. Sér. A. 1979. V. 289. № 11. P. 583-585. 12. Черкашин В. П. // УМН. 1995. Т. 50. № 2. С. 225-226. 